\title{
Hybrid BOTDA/FBG sensor for discrete dynamic and distributed static strain/temperature measurements
}

\author{
Tiziano Nannipieri ${ }^{\mathrm{a}, *}$, Mohammad Taki ${ }^{\mathrm{a}}$, Farhan Zaidi ${ }^{\mathrm{a}}$, Alessandro Signorini ${ }^{\mathrm{a}}$, Marcelo A. Soto ${ }^{\mathrm{a}, \mathrm{b}}$, \\ Gabriele Bologninic ${ }^{c}$, Fabrizio Di Pasquale ${ }^{\mathrm{a}}$. \\ ${ }^{\text {a }}$ Scuola Superiore Sant'Anna, via G. Moruzzi 1, 56124 Pisa, Italy. \\ ${ }^{\mathrm{b}}$ Current address EPFL Swiss Federal Institute of Technology, Institute of Electrical Engineering, \\ SCI-STI-LT, Station 11, 1015 Lausanne, Switzerland \\ ${ }^{c}$ Consiglio Nazionale delle Ricerche, IMM Institute, via P. Gobetti 101, 40129 Bologna, Italy \\ *t.nannipieri@sssup.it
}

\begin{abstract}
We propose and experimentally demonstrate a hybrid fiber optic sensing technique that effectively combines Brillouin optical time-domain analysis and a time-domain multiplexing interrogation technique for Fiber Bragg Gratings (FBGs). The highly-integrated proposed scheme employs broadband apodized low-reflectivity FBGs with a single optical source and a shared receiver block, allowing for simultaneous measurements of distributed static and discrete dynamic temperature and strain, over the same sensing fiber.
\end{abstract}

Keywords: Brillouin scattering, Fiber Bragg Gratings, fiber optic sensors, temperature sensing, strain sensing.

\section{INTRODUCTION}

During the last years, distributed optical fiber sensing based on stimulated Brillouin scattering (SBS) has become an interesting approach for temperature and strain sensing in many industrial applications. In particular, distributed sensing with meter and sub-meter spatial resolution along several tens of $\mathrm{km}$ has been demonstrated using Brillouin optical timedomain analysis (BOTDA) technique [1]. Even though the sensing capabilities provided by BOTDA systems are attractive in many applications, the relatively long measurement time (of the order of a few minutes) makes this technology mainly suitable for static measurements, limiting its potential range of applications. Although the distributed information provided by BOTDA sensing is essential for static strain and temperature monitoring, there are several applications, such as for example in industrial Oil\&Gas production plants monitoring, which also require dynamic strain measurements at specific critical points, adding in this way crucial information on the structure integrity (e.g. vibrations). Distributed sensing based on either spontaneous Brillouin scattering (SpBS) or SBS has been recently combined with point based Fiber Bragg Grating (FBG) sensors $[3,4]$. In particular, BOTDA technique has been combined with wavelength-division-multiplexed FBG point sensors, using different demodulation techniques for distributed and discrete sensing, as well as distributed Raman amplification, increasing cost and complexity of the whole sensor system [4].

In this paper we propose a highly-integrated hybrid sensing system that effectively combines the advantages of both standard BOTDA and in-line time-division-multiplexed (TDM) FBG-based sensing, allowing for simultaneous dynamic discrete strain measurements and distributed (static) strain/temperature sensing using a single optical fiber, a common pulsed narrowband optical source and a shared receiver unit.

\section{THEORY}

In BOTDA systems, a pulsed pump and a counter-propagating continuous-wave (CW) probe light interact with acoustic phonons generated into the sensing fiber by SBS [1]. The maximum energy transfer from the pump to the probe (i.e. Brillouin amplification) takes place at every fiber location whenever the frequency separation among the optical waves equals the local acoustic frequency in the fiber, the so-called Brillouin frequency shift (BFS), which is a temperatureand strain-dependent parameter. By sweeping the pump-probe frequency difference, the Brillouin Gain Spectrum (BGS) can be reconstructed and the BFS can be estimated, providing information about the temperature and strain variations along the whole sensing fiber. Unfortunately, the BGS reconstruction technique requires typical acquisition times of the order of a few minutes, limiting then the application of the standard BOTDA technique to static temperature/strain 
sensing only. When dynamic strain measurements in specific critical points are required in addition to the distributed static temperature/strain measurement along the whole sensing fiber, more complex techniques are required. A typical example consists in fire detection and vibrations measurement in large civil and industrial structures.

On the other hand, FBG-based dynamic sensing is a well-known technique [2] which exploits the Bragg wavelength $\left(\lambda_{B}\right)$ dependence on temperature variation $(\Delta \mathrm{T})$ and strain $(\varepsilon)$, given by the formula:

$$
\Delta \lambda_{B}=\lambda_{B}\left[(\alpha+\gamma) \Delta T+\left(1-p_{e}\right) \varepsilon\right]
$$

where $\Delta \lambda_{B}$ is the variation of Bragg wavelength, $\alpha$ and $\gamma$ are the thermal expansion and thermo-optic coefficients respectively, and $p_{e}$ is the effective photo-elastic constant of the fiber material.

The basic operating principle of the FBG interrogation technique exploited in this work, for a dynamic strain estimation and compatible with simultaneous BOTDA measurements, is illustrated in Fig.1; the technique employs a single pulsed narrowband pump laser and a pair of Gaussian apodized FBGs with low reflectivity and broadband spectrum at each sensing point. The two FBGs are placed close to each other, with a short connection fiber (few meters spatial separation) allowing for detection of FBG response through reflectometry using the same pump as in BOTDA (1 m minimum spatial resolution); the FBG pair is placed within a packaged coiling in which both gratings L-FBG (left) and R-FBG (right) are attached to the structure of interest, and therefore made simultaneously sensitive to both strain and temperature.

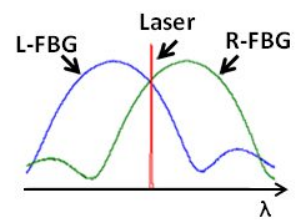

Figure 1. Optical spectrum of the proposed dynamic strain interrogation technique

Since the two reflected spectra of the FBGs within the same sensing point are symmetrically shifted with respect to the central wavelength of the laser source, using the ratio of back-reflected intensities from both FBGs can provide immunity to fiber losses and laser power fluctuations. More specifically, an interrogation function $\rho\left(\Delta \lambda_{B}\right)$ has been used, given by:

$$
\rho\left(\Delta \lambda_{B}\right)=\ln \left(\int_{z_{L-F B G}}^{Z_{L-F B G}+\Delta_{Z}} I_{L R-F B G}\left(\Delta \lambda_{B}, \xi\right) d \xi\right)-\ln \left(\int_{Z_{R-F B G}}^{Z_{R-F B G}+\Delta_{Z}} I_{L R-F B G}\left(\Delta \lambda_{B}, \xi\right) d \xi\right)
$$

where $\xi$ is the fiber distance, $I_{L R-F B G}\left(\Delta \lambda_{B}, \xi\right)$ is the back-reflected intensity trace from FBGs, $Z_{L-F B G}\left(Z_{R-F B G}\right)$ is the location of L-FBG (R-FBG), and $\Delta_{Z}$ is the spatial extent of FBG response for given pulse conditions in BOTDA pump. Considering the Gaussian shape of the used FBG, the interrogation function can be assumed approximately linear for typical $\Delta \lambda_{\mathrm{B}}$ excursion of practical applications.

\section{EXPERIMENTAL SETUP}

The schematic setup of the proposed hybrid BOTDA-FBG sensor is shown in Fig. 2. In this scheme, the light provided by an external cavity laser (ECL, $0 \mathrm{dBm}$ output power at $1549.8 \mathrm{~nm}$ ) is split into two branches using a $3 \mathrm{~dB}$ splitter. One of the branches is employed to generate a pulsed signal which is used as Brillouin pump as well as to interrogate the FBGs-based discrete sensing points placed in cascade along the sensing fiber.

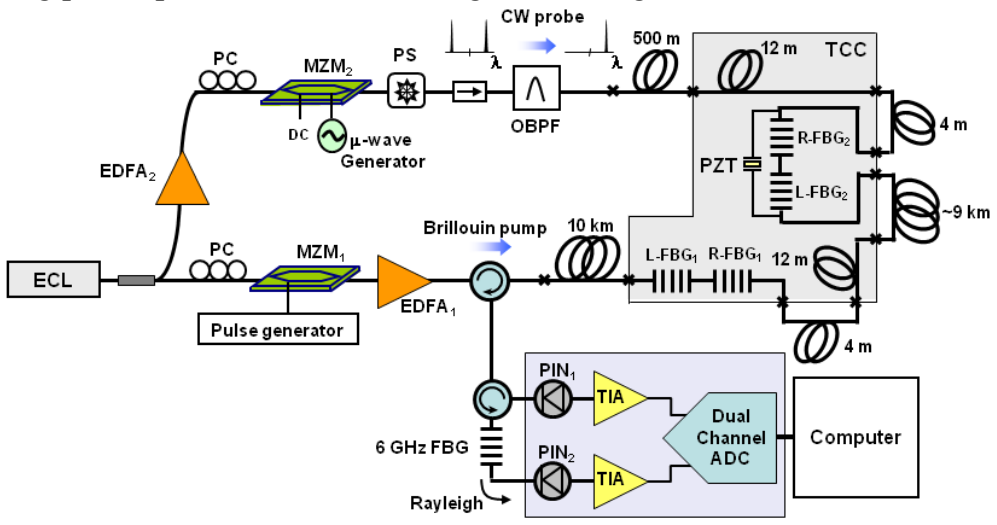

Figure 2. Experimental setup. 
A polarization controller (PC) and a Mach-Zehnder modulator $\left(\mathrm{MZM}_{1}\right)$, driven by a pulse generator, are used to modulate the intensity of $\mathrm{CW}$ light with $10 \mathrm{~ns}$ pulses (providing $\sim 1 \mathrm{~m}$ spatial resolution) at a repetition rate of $5 \mathrm{kHz}$. An Erbium-Doped fiber amplifier $\left(\mathrm{EDFA}_{1}\right)$ is used to obtain a peak pulse power of $20 \mathrm{dBm}$ at the fiber input. In the other branch, the $\mathrm{CW}$ light from the ECL is amplified by $\mathrm{EDFA}_{2}$ and then modulated through a second Mach-Zehnder modulator $\left(\mathrm{MZM}_{2}\right)$ with a microwave signal in order to generate a two-sideband probe signal (suppressed carrier). A polarization scrambler (PS) is used to depolarize the probe signal and to avoid polarization-induced fluctuations in the Brillouin gain. A narrow optical band-pass filter (OBPF, bandwidth $<100 \mathrm{pm}$ ) is used to select the low-frequency probe sideband only (Stokes frequency), thus filtering out ASE noise, the residual suppressed carrier and the high-frequency probe sideband (anti-Stokes component). Light pulses are coupled into a $\sim 20 \mathrm{~km}$ standard SMF through an optical circulator which is also used to extract both the probe signal (used in BOTDA sensing) and the back-reflected component at the pump frequency (used for FBGs interrogation). Both light components are coupled to a shared receiver stage, composed of an optical circulator and a narrowband FBG (6 GHz bandwidth), which separates the probe and the backreflected pump light components onto different ports of a single multi-channel photoreceiver (employing PIN photodiodes, $125 \mathrm{MHz}$ analog bandwidth).

\section{RESULTS}

A pair of in-line FBG-based sensing points have been placed at $\sim 10 \mathrm{~km}$ and $\sim 19 \mathrm{~km}$ distances along the sensing fiber, both pairs within a temperature controlled chamber (TCC); each sensing point consist of a pair of spatially close Gaussian-apodized FBGs ( $\sim 2 \mathrm{~m}$ separation, $2.5 \mathrm{~nm}$ bandwidth, $1 \%$ peak reflectivity), which are centered at two different wavelengths (L-FBG at $1548.65 \mathrm{~nm}$ and R-FBG at $1550.85 \mathrm{~nm}$ ). The FBG pair at $19 \mathrm{~km}$ sensing point has been glued to a piezoelectric actuation stage for dynamic strain sensing assessment; the FBG-response of this sensing point, located near fiber end, has been first statically characterized as a function of strain and temperature, setting the PZT actuation stage and TCC at different values and measuring the time-domain traces of the back-reflected lights from both FBGs; these are shown in Fig. 3(a). For a proper FBG characterization, 1000 traces have been averaged at each TCC temperature and strain value, and the interrogation function has then been calculated. Figure 3(b) shows the observed behavior (highly linear as expected by theory) of the interrogation function versus temperature and strain comparing linear fitting (solid line) with experimental data (square dots). The same behavior was observed in the FBG pair at 10 $\mathrm{km}$.
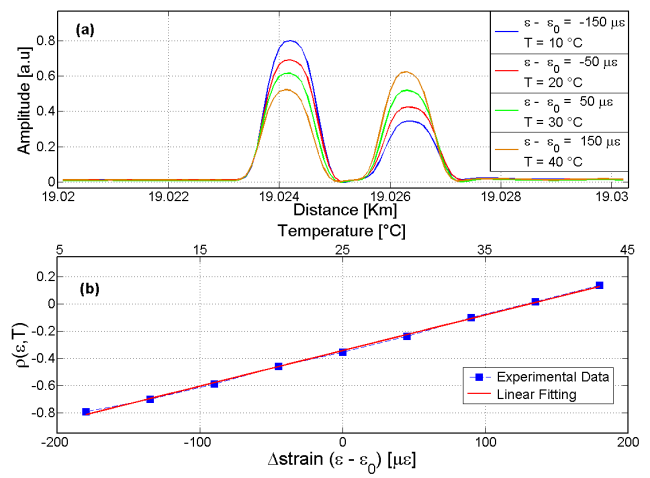

Figure 3. (a) Reflected pulse intensity from both FBGs at different strain and TCC temperatures, and (b) characterization of the interrogation function against applied strain and temperature.

In order to investigate the compatibility of simultaneous time-domain FBGs interrogation and BOTDA technique, the BGS along the fiber length (whole fiber placed at room temperature, $25^{\circ} \mathrm{C}$ ) has been reconstructed and is shown in Fig. 4. We have verified that the BGS follows a uniform Lorenztian profile and that no distortion is present along the 20 $\mathrm{km}$ fiber length. Furthermore, we verified that the two sensing points placed at $\sim 10$ and $\sim 19 \mathrm{~km}$ distances are not affecting the Brillouin measurement, thanks to the low FBGs reflectivities leading to negligible distortions in the BGS. In particular Fig. 5 shows the measured temperature after the second FBG sensing point with $12 \mathrm{~m}$ of fiber at two different TCC temperatures $5^{\circ} \mathrm{C}$ and $45^{\circ} \mathrm{C}$, clearly demonstrating the negligible impact of the FBGs back-reflected light (which strongly depends on temperature) on the measurement. Due to the very low reflectivity values, we expect that our BOTDA measurement technique will be compatible with a large number of discrete sensing points. The resolution in terms of BFS has been estimated by calculating the standard deviation of the measured BFS distribution along the fiber length, resulting to be $2.4 \mathrm{MHz}$ near the fiber end $(\sim 20 \mathrm{~km})$ and corresponding to a temperature and strain resolution of $2.4^{\circ} \mathrm{C}$ and $48 \mu \varepsilon$ respectively. 


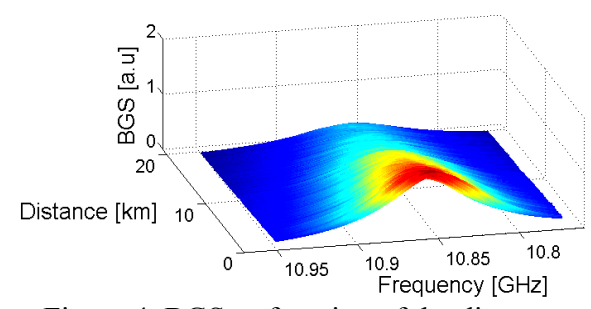

Figure 4. BGS as function of the distance

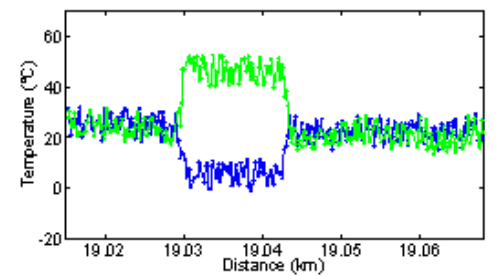

Figure 5. Temperature profile as a function of distance at the fiber end, measured with $5^{\circ} \mathrm{C}$ and $45^{\circ}$ temperature changes of the TCC.

Finally, dynamic strain measurements have been assessed by applying a sinusoidal strain waveform (350 $\mu \varepsilon$ peak-topeak) through the PZT. It is worth mentioning that, although strain and temperature variations are mapped to the same interrogation function values, dynamic strain variations can be extracted using frequency-domain high-pass filtering thus removing the static component of the signal. Figure 6 shows an example of dynamic strain acquisition at $25{ }^{\circ} \mathrm{C}$ and $0.25 \mathrm{kHz}$. In particular, Fig. 6(a) illustrates the measured dynamic strain trace, which is in good agreement with the (known) applied strain waveform; Fig. 6(b) shows the normalized fast Fourier transform (FFT) of the measured trace from which we can see that the fundamental component is easily identified among other (smaller) spurious spectral components. The dynamic strain resolution was estimated to be $24.2 \mathrm{n} \varepsilon /(\mathrm{Hz})^{1 / 2}$ at $0.25 \mathrm{kHz}$.
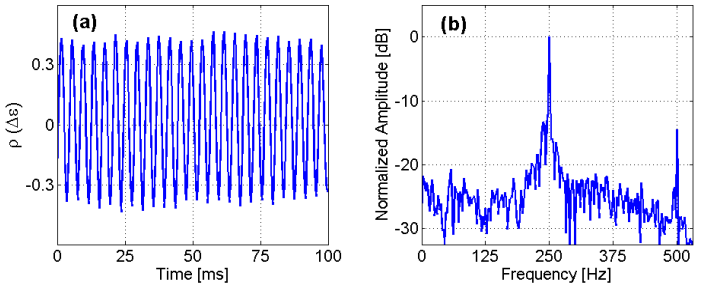

Figure 6. Dynamic strain measurements at $0.25 \mathrm{kHz}$. (a) Time-domain trace and (b) fast Fourier transform.

In conclusion, a hybrid BOTDA/FBG sensor system using a highly-integrated interrogation unit (sharing a narrowband pulsed source, the sensing fiber and the receiver stage) has been implemented. Experimental results show concurrent distributed sensing, with temperature (strain) resolution of $2.4{ }^{\circ} \mathrm{C}(48 \mu \varepsilon)$ throughout $\sim 20 \mathrm{~km}$ fiber length, and dynamic sensing with discrete FBG dynamic strain resolution of $24.2 \mathrm{n \varepsilon} /(\mathrm{Hz})^{1 / 2}$ at $0.25 \mathrm{kHz}$, enabling the use of such a simple and effective hybrid technique in many applications where distributed static and discrete dynamic measurements are simultaneously required.

\section{REFERENCES}

[1] Taki, M., Soto, M. A., Bolognini, G., and Di Pasquale, F., "Long-range distributed strain and temperature sensing with 40-cm spatial resolution based on DPP-BOTDA employing optical pre-amplification and Simplex coding", Optical Fiber Communication Conference, paper OTuL2, Los Angeles, US, 6-10 March 2011.

[2] Kersey, A. D., Davis, M. A., Park, H. J., Leblan, M., and Koo, K. P., "Fiber Grating Sensors," J. Lightwave Technol. 15, 1142-1463 (1997).

[3] Sun, A., Semenova, Y., Farrell, G., Chen, B., Li, G., and Lin, Z., "BOTDR integrated with FBG sensors array for distributed strain measurements" Electron. Lett. 46, 66-67 (2010).

[4] Fernandez, M., Olier, D., Zornoza, A., Perez, R., Diaz, S., Elosua, C., Bariain, C., Loayssa, A., and Lopez-Amo Sainz, M. "46-km Long Range Raman amplified Hybrid Double-Bus Network with point and distributed Brillouin sensors” IEEE Sens. J. 12, 184-188 (2012). 